Invited paper for the Special Issue of Journal of Materials Science

Special Issue Title: "Nanostructured Materials and Mechanical Behavior"

\title{
Dynamics of Nanoscale Grain-Boundary Decohesion in Aluminum by Molecular-Dynamics Simulation
}

\author{
V. Yamakov ${ }^{1 *}$, E. Saether ${ }^{2}$, D. R. Phillips ${ }^{3}$, and E.H. Glaessgen ${ }^{2}$ \\ ${ }^{1}$ National Institute of Aerospace, Hampton, VA 23666, USA \\ 2 NASA Langley Research Center, Hampton, VA 23681, USA \\ ${ }^{3}$ Lockheed Martin Space Operations, Hampton, VA 23681, USA
}

The dynamics and energetics of intergranular crack growth along a flat grain boundary in aluminum is studied by a molecular-dynamics simulation model for crack propagation under steady-state conditions. Using the ability of the molecular-dynamics simulation to identify atoms involved in different atomistic mechanisms, it was possible to identify the energy contribution of different processes taking place during crack growth. The energy contributions were divided as: elastic energy - defined as the potential energy of the atoms in fcc crystallographic state; and plastically stored energy - the energy of stacking faults and twin boundaries; grain-boundary and surface energy. In addition, monitoring the amount of heat exchange with the moleculardynamics thermostat gives the energy dissipated as heat in the system. The energetic analysis indicates that the majority of energy in a fast growing crack is dissipated as heat. This dissipation increases linearly at low speed, and faster than linear at speeds approaching $1 / 3$ the Rayleigh wave speed when the crack tip becomes dynamically unstable producing periodic dislocation bursts until the crack is blunted.

\footnotetext{
*Corresponding author: Tel.: +757-864-2850; fax: +757-864-8912.

E-mail address: yamakov@,nianet.org (V. Yamakov)
} 
Invited paper for the Special Issue of Journal of Materials Science Special Issue Title: "Nanostructured Materials and Mechanical Behavior"

\section{Introduction}

Intergranular fracture is the governing process for mechanical failure in nanocrystalline metals [1]. Intergranular crack propagation is strongly influenced by the specifics of the grain boundary (GB) interface, the structure of which still poses many unresolved issues [2]. The GB volume at the front of the approaching crack tip undergoes structural changes, which, in turn, affect the crack propagation. The presence of crystals with two different crystallographic orientations, containing slip systems oriented differently to the crack plane on both sides of the crack, is also another complicating factor which has to be taken into account. The presence of the GB also affects the energetics and dynamics of the intergranular crack propagation. While there are a large number of studies on the structural processes at the crack tip (some of them, related to intergranular cracks will be discussed below), little is known on the energy transformations accompanying these processes, which defines the crack growth resistance.

The propagation of a crack involves a substantial amount of energy to be consumed or dissipated. The energy balance has a governing role in the process of fracture. In its simplest form, for a perfectly brittle material, the energy balance is expressed by Griffith's equation [3] as

$$
G=2 \gamma_{s}
$$

where $G$ is the energy release rate associated with crack extension, and $\gamma_{\mathrm{s}}$ is the free energy of the newly created surface on each side of the crack. For ductile materials, Orowan [4] and Irwin [5] extended Eq. (1) to include the plastic energy per unit area $\gamma_{\mathrm{pl}}$ dissipated through various irreversible processes in the plastic zone surrounding the crack tip [6]

$$
G=2 \gamma_{s}+\gamma_{p l}
$$

In the case of intergranular fracture, the energy of the GB $\gamma_{\mathrm{GB}}$ has an additional contribution to the balance 
Invited paper for the Special Issue of Journal of Materials Science

Special Issue Title: "Nanostructured Materials and Mechanical Behavior"

$$
G=2 \gamma_{s}+\gamma_{p l}-\gamma_{G B},
$$

indicating that it is easier to grow a crack along a GB interface than through a perfect crystal. A systematic analysis on the relation between GB crystallography, GB energy, and GB cleavage properties was performed by Wolf [7] using molecular-dynamics (MD) simulations of copper and gold. In this study [7], a large representative set of symmetric tilt and twist GBs were considered according to two characteristic parameters, the twist and tilt angle $(\varphi, \theta)$, and their cleavage energies, $E_{c l}$, defined by analogy with Eq. (3) as

$$
E_{c l}=2 \gamma_{s}-\gamma_{G B}(\varphi, \theta)
$$

were estimated. As a result, a structure-energy correlation for all symmetric GBs in an fcc metal was derived and related to the inter-granular fracture properties. The role of plasticity was not considered in ref. [7], but investigations on dislocation nucleation from the crack tip as a function of the GB crystallography and structure were performed in a series of subsequent works [8-10]. Intergranular crack induced plasticity in nanocrystalline metals was studied in [10-12]. The decohesion strength of a GB and its dependence on impurities was studied in [13-16]. The dependence of the fracture stress and strain of the GB interface on the atomic disorder due to the presence of vacancies and interstitials, has also been recently investigated [17].

One approach to analyze the plasticity contribution to crack resistance has been suggested by Stampfl and Kolednik [18]. The total plastically deformed volume is divided into several characteristic regions, according to the distance from the crack surface, and the energy contribution of each region to $\gamma_{\mathrm{pl}}$ is determined. The idea is based on experimental findings that crack growth resistance often depends on the geometry and the size of the specimen, thus implying that different volume regions surrounding the crack contribute differently to $G$ [18]. 
Invited paper for the Special Issue of Journal of Materials Science Special Issue Title: "Nanostructured Materials and Mechanical Behavior"

A more fundamental physics based approach to study crack resistance is presented in this paper. The energy dissipation at the crack tip is determined through identifying the underlying physical process and quantifying individual contributions during crack propagation. A Molecular Dynamics (MD) simulation of a crack growing along a high-angle $\sum 99<110>$ tilt GB in aluminum is used to interrogate the atomic processes associated with the crack tip. MD simulations have the advantage that they can capture the dynamics of crack propagation at an atomic scale. By integrating the Newtonian equations of motion to calculate atomic trajectories, MD simulations can give invaluable information not only on the structural changes in the system, but also on the energetics and dynamics of the underlying processes. By using special techniques in identifying atoms in different structural states (specified further in the text), the energy spent for crack propagation can be separated into different categories according to its participation in certain physical processes. As a result, a complete picture of the energy balance of the crack propagation, revealing a variety of energy transformations can be obtained. Such an analysis could help in better understanding the energetics of crack propagation in nanocrystalline fcc metals and in other materials, where intergranular fracture is a dominant failure mechanism.

This paper is constructed as follows: The simulation approach is described in Section 2. The crack growth dynamics is presented in Section 3. A detailed analysis of the energy dissipated in the process of crack propagation is given in Section 4. The main conclusions of this study are outlined in Section 5.

\section{The simulation approach}

The simulation approach used in this study is based on a MD simulation model of crack propagation under time-independent, or steady-state conditions through a flat grain boundary in 
Invited paper for the Special Issue of Journal of Materials Science Special Issue Title: "Nanostructured Materials and Mechanical Behavior"

aluminum modeled by the interatomic potential of Mishin et al. [19]. The simulation is performed at a temperature of $100 \mathrm{~K}$ to suppress GB and surface diffusion and facilitate brittle fracture. The temperature is maintained by using a molecular-dynamics Nose-Hoover thermostat [20]. The MD simulation set up follows the known analytical model for steady-state crack propagation [21] under constant strain conditions and has the configuration schematically shown in Fig. 1. With periodic boundary conditions in all directions, the model represents an aluminum multilayer system of alternating sets of thick and thin crystalline layers separated by four flat GBs. The two broad layers, marked as "Crystal I" and "Crystal II", form a bicrystalline system with a flat GB in the middle, through which the crack propagates. The crystallographic orientations of Crystal I and Crystal II are presented in Fig. 2 representing a snapshot of an enlarged part of the simulation system around the GB after relaxation. In the imposed coordinate system of the model, the orientation of Crystal I is: $\left(x:\left[\begin{array}{lll}7 & \overline{7} & \overline{10}\end{array}\right] ; \mathrm{y}:\left[\begin{array}{lll}5 & \overline{5} & 7\end{array}\right] ; \mathrm{z:}:\left[\begin{array}{lll}1 & 1 & 0\end{array}\right]\right)$, and the orientation of Crystal II is: (x:[ $\left.\begin{array}{lll}7 & \overline{7} & -\overline{0}\end{array}\right]$; $\mathrm{y}:\left[\begin{array}{ccc}\overline{5} & 5 & \overline{7}\end{array}\right]$; z: $\left.\left[\begin{array}{lll}1 & 1 & 0\end{array}\right]\right)$ (see Fig. 2). In this way, Crystal II is a mirror image of Crystal I relative to the crystallographic plane $\left\{\begin{array}{lll}5 & 5 & 7\end{array}\right\}$, which becomes the plane of the GB between them. The GB thus formed is a $<110>\sum 99$ symmetric tilt GB. Its atomic structure in Al agrees well with that determined through transmission electron microscopy (TEM) investigations [22]. This is a high-angle grain boundary (tilt angle of $89.42^{\circ}$ ) with a large excess (i.e., above the perfect crystal) energy, $\gamma_{\mathrm{GB}}=0.60 \pm 0.05$ $\mathrm{J} / \mathrm{m}^{2}$, estimated here for a relaxed structure at $\mathrm{T}=100 \mathrm{~K}$. The high excess GB energy facilitates its decohesion [7]. The surface energy of the GB plane $\gamma_{\mathrm{s}}$ at $100 \mathrm{~K}$ is estimated in this study as $\gamma_{\mathrm{s}}$ $=0.952 \pm 0.010 \mathrm{~J} / \mathrm{m}^{2}$ and is in good agreement with experimental data for the Al surface energy.

The two smaller layers, Absorbing Layer I and Absorbing Layer II, on both sides of the bicrystalline system (Fig. 1) have the same crystallographic orientations as Crystal II and Crystal 
Invited paper for the Special Issue of Journal of Materials Science Special Issue Title: "Nanostructured Materials and Mechanical Behavior"

I, respectively. Consequently, the GBs formed by these layers are of the same crystallographic type as the GB between Crystal I and Crystal II. The purpose of these layers in the simulation is to serve as absorbers for the phonon waves [15] generated from the crack tips preventing their reentrance back into the system through the imposed periodic boundary conditions, which may influence the crack propagation. The wave absorption is performed by applying a damping friction coefficient to the atoms in the absorbing layers. This damping effectively takes energy from the system and decreases the temperature of the layers. The applied Nose-Hoover thermostat [20] compensates for the absorbed energy by supplying an additional amount of energy to keep the average temperature constant at $100 \mathrm{~K}$. The sum of the energy taken by the absorbing layers and by the thermostat would make the total heat $\Delta Q$ released by the system in the process of crack growth. In addition to absorbing phonon waves, the GBs created between these layers and Crystals I and II act as sinks for dislocations that may be emitted and propagate from the crack tips during crack growth. In this way, the negative effect of the periodic boundary conditions in the $y$-direction creating periodic images of all crack-tip disturbances and influencing the crack propagation is suppressed.

The interaction between individual atoms in the system is presented by a many-body embedded-atom method (EAM) potential of Mishin et al. [19] fitted to give the correct zerotemperature lattice constant, $\mathrm{a}_{\mathrm{o}}=4.05 \AA$, elastic constants, cohesive energy, vacancy formation energy, etc. Of particular importance for the simulation of fracture and dislocation plasticity is the close fit of the potential to the experimentally measured surface and stacking-fault energies. Potential-dependent parameters that will be needed in this study are the relaxed stable stackingfault energy, $\gamma_{\mathrm{sf}}=0.146 \mathrm{~J} / \mathrm{m}^{2}$, the unstable stacking-fault energy, $\gamma_{\mathrm{us}}=0.168 \mathrm{~J} / \mathrm{m}^{2}$, as defined in 
Invited paper for the Special Issue of Journal of Materials Science Special Issue Title: "Nanostructured Materials and Mechanical Behavior"

ref. [19], and the unstable twinning energy, $\gamma_{\mathrm{ut}}=0.210 \pm 0.010 \mathrm{~J} / \mathrm{m}^{2}$, estimated here according to the method described in ref. [23].

The system thickness $h$ in the $z$-direction equals only 10(2 20 ) crystallographic planes (accounting for the symmetry of the fcc lattice), or $h=10 \sqrt{2} / 2 \mathrm{a}_{\mathrm{o}} \approx 2.9 \mathrm{~nm}$. This thickness is more than four times larger than the range of the interatomic potential, $r_{\mathrm{c}}=1.55 \mathrm{a}_{\mathrm{o}}=0.628 \mathrm{~nm}[19]$, which is sufficient to prevent interference of the atoms with their periodic images and to preserve the local three-dimensional (3D) physics in the system. The small thickness in the $z$-direction allows the system size in the $x$ - and $y$-directions to extend up to $21<7710>\mathrm{a}_{\mathrm{o}} \approx 120 \mathrm{~nm}$, and $24<557>\mathrm{a}_{\mathrm{o}} \approx 97 \mathrm{~nm}$, respectively (see Fig 1 and Fig. 2), while limiting the number of simulated atoms to $1,994,000$, allowing the simulation to be carried out on a modest Beowulf cluster.

As the dislocation activity is expected to be very important in this study, the choice of the [1 $10]$ direction as a columnar axis for this quasi-two dimensional set-up ensures that two slip planes (the $\left(\begin{array}{lll}\overline{1} & 1 & 1\end{array}\right)$ and $\left(\begin{array}{lll}1 & \overline{1} & 1\end{array}\right)$ planes) with a total of six slip systems are presented in each crystal $[24,25]$. The existence of two slip planes allows for a variety of dislocation interactions and cross slip events to take place [24,25] resembling closely a full $3 \mathrm{D}$ environment, the main constraint being that the dislocation lines of all possible dislocations have to be straight lines parallel to the columnar direction. The implication of this constraint affects mostly the stress barrier for dislocation nucleation making it slightly higher for a straight dislocation, compared to a dislocation loop in a fully 3D system [26]. While neglecting the effects of curvature is not expected to qualitatively change the fracture mechanism, it may affect the process quantitatively in terms of slightly decreasing peak stress ${ }^{*}$ and work of decohesion.

\footnotetext{
* The stress to nucleate a dislocation from a grain boundary in a nanocrystalline columnar simulated microstructure was found to be $2.3 \mathrm{GPa}$ [24], while in a fully $3 \mathrm{D}$ microstructure of the same material it was found to be less than $2.0 \mathrm{GPa}[26]$.
} 
Invited paper for the Special Issue of Journal of Materials Science

Special Issue Title: "Nanostructured Materials and Mechanical Behavior"

After equilibration at zero constant pressure using the Parrinello-Rahman constant-stress simulation [27], the system is loaded hydrostatically in tension, i.e.,

$$
\sigma_{x x}=\sigma_{y y}=\sigma_{z z}=\sigma
$$

and is dynamically equilibrated at this constant stress. After establishing equilibrium between the strain in the system and the applied external stress, the system size in all three dimensions is fixed under constant strain conditions.

The transition from a constant stress to a constant strain simulation transforms the volume fluctuations that are always present in a finite system under thermodynamic equilibrium into stress fluctuations. Thus, further equilibration at constant strain is necessary to smooth out these fluctuations. The simulation then proceeds under this constant strain - constant temperature condition. Although the simulations are carried out under constant strain, for convenience in presentation, the various analyses will reference the value of prestress that corresponds to a particular value of prestrain.

The crack in the system is nucleated by screening (preventing) the atomic interactions between atoms at both sides of the GB plane between Crystal I and Crystal II along a region of length $l_{\mathrm{o}}$. The crack starts growing if $l_{\mathrm{o}}$ is larger than the critical Griffith length $L_{g}$ defined when the energy spent to create the upper and lower crack surface $2 \gamma_{\mathrm{s}}$ minus the energy gained by destroying the GB $\gamma_{\mathrm{GB}}$ is equal to the released strain energy $-d U / d l$, per length $l$,

$$
2 \gamma_{\mathrm{s}}-\gamma_{\mathrm{GB}}=-d U / d l
$$

An estimate of $L_{g}$ is made by calculating $d U / d l$ as a function of $l$ and $\sigma$. This calculation is performed by using an anisotropic elastic finite element model of the elastic equivalent for the MD system, as described in ref. [28]. $L_{g}$ sets the minimum size to grow a crack in the system. In order to simulate a sufficient crack growth length, $L_{g}$ must be at least one order of magnitude 
Invited paper for the Special Issue of Journal of Materials Science Special Issue Title: "Nanostructured Materials and Mechanical Behavior"

smaller than the system size. For this reason and to reflect the existing periodicity of the equilibrated GB structure induced by the lattice of the two joined crystals [22], the screened region length is set equal to the crystallographic period in the $x$-direction, or $l_{0}=\left\langle\begin{array}{lll}7 & 7 & 10\end{array}\right\rangle a_{0}=5.7 \mathrm{~nm}$. The performed calculations for $L_{g}$ satisfying Eq. (6) give $L_{g}<l_{\mathrm{o}}$ for $\sigma>3.5 \mathrm{GPa}[28]$. Thus, the values of the prestress in this study were set to $\sigma=3.5,3.75,4.0$, and 4.25 GPa. Crack growth has been observed even at $\sigma=3.5 \mathrm{GPa}$ because the interaction range of the many body potential increases the effective length of the screened region as all the atoms within the interaction range of the screened atoms are also affected by the screening and make the initial crack length effectively larger than prescribed.

These very high prestress values, imposed by the severe system size limitations in the MD simulation, are larger than the yield strength of the material and would have caused strong plasticity effects not related to the crack, such as spontaneous dislocation nucleation from the GBs [24-26]. Applying triaxial hydrostatic stress (Eq. (5)) eliminates these undesirable plasticity effects.

The identification of various structural defects including dislocations, twins, stacking faults, etc., appearing around the growing crack is important for understanding the various deformation mechanisms in the system. A procedure for atom identification based on the atom's coordination number and on the common-neighbor-analysis (CNA) technique $[29,30]$ is used. The technique makes it possible to identify atoms in fcc and hcp states. Layers of hcp atoms in an fcc lattice are formed at stacking faults and twin boundaries [31] and can be used successfully for visualizing the ongoing dislocation processes in fcc crystals [32]. Atoms that are not identified in an fcc or hcp state are considered to be in a non-crystalline state and indicate the presence of GBs or dislocation cores. In addition, atoms that have lost more than $1 / 3$ of their neighbors inside the 
Invited paper for the Special Issue of Journal of Materials Science

Special Issue Title: "Nanostructured Materials and Mechanical Behavior"

interaction range of the potential are considered surface atoms. Under this convention, the thermalized structure of a $\langle 110\rangle \Sigma 99$ symmetric tilt GB, shown in Fig. 2, appears as quasiperiodic, with a regular pattern of hep atoms immersed in a disordered layer with few distributed vacancies, identified by the appearance of few isolated surface atoms inside the GB region. As will be shown later in this paper, classifying atoms in this way presents a unique possibility to distinguish and quantify the various atomic processes occurring at the crack tip, and to identify their contribution to the work of decohesion for the GB interface.

\section{Dynamic crack growth along the $\Sigma 99$ grain boundary}

The process of intergranular crack growth at $3.75 \mathrm{GPa}$ prestress is monitored through the series of snapshots in Fig. 3. The identified hcp atoms form the stacking faults and twin boundaries visualized as dark straight lines (2) in Figs. 3(a-d)) passing through the interiors of the two crystals. The disordered atoms form the GB seen as a thick black line dividing the two crystals (1) in Fig. 3(a)), the dislocation cores of the twinning dislocations, seen along the twin boundaries (3) in Figs. 3(b-d)), and the cores of the slip dislocations found in the crystals' interior (5) in Fig. 3(i)). The wavy patterns in the crystal regions are Moire patterns produced by the overlapping of the elastically deformed atomic lattice with the regular pixel grid of the digital image. In a way, these patterns make visible the strain patterns and phonon waves in the crystal.

The MD simulation is used to determine the mechanisms of formation and crack growth from initiation at $t=0 \mathrm{ps}$ through $t=104 \mathrm{ps}$. As seen in Fig. 3, the mechanisms of crack growth in the $-x$ direction and $+x$ direction are entirely different.

As early as 4 ps after initiation $(t=4 \mathrm{ps})$, a nanotwin starts to form at the crack tip propagating in the $-x$ direction (2) in Fig. 3(a)). The nanotwin grows (2) in Figs. 3(b, c)) by continuous emission of a series of twinning dislocations from the crack tip (3) in Figs. 3(b, c)) 
Invited paper for the Special Issue of Journal of Materials Science Special Issue Title: "Nanostructured Materials and Mechanical Behavior"

[31]. At $t=12$ ps after the crack initiation, a second nanotwin at the same crack tip, but on the opposite side of the GB is nucleated (see both identifiers (3) in Fig. 3(c)) and proceeds to grow symmetrically with the path of the first nanotwin (2) and (3) in Figs. 3(c, d)). Both nanotwins continue to grow in a symmetric fashion as shown in the remaining snapshots (Figs. 3(e-i)).

Conversely, the crack propagation in the $+x$ direction takes place through a continuous process of void formation and void coalescence (4) in Figs. 3(d-i)). Compared to the crack growth in the $-x$ direction, where the crack tip is effectively blunted by the nanotwins, the crack growth in the $+x$ direction is very rapid. In spite of its intensity, this growth has not produced any dislocation emission for $76 \mathrm{ps}$ after the crack initiation (Figs. 3(a-g)). In the time interval between 76 and 104 ps (see Figs. 3(g) and 3(i)), the crack tip suddenly emits a burst of dislocations (5) in Fig. 3(i)) and the growth in the $+x$ direction stops. Further simulation shows no more crack propagation or growth of the nanotwins, indicating that the crack has reached equilibrium at a length close to $40 \mathrm{~nm}$. This asymmetric crack growth in the $+x$ and $-x$ directions is due to the specific orientation of the $\{111\}$ slip planes on both sides of the GB interface forming different inclination angles with the propagation directions of the two crack tips. The observation is in agreement with the Rice [33] and Tadmor-Hai [23] criteria for twinning vs. cleavage at the crack tip, as demonstrated in ref. [28].

The equilibrium states for the other three cases of prestress, $\sigma=3.5,4.0$, and $4.25 \mathrm{GPa}$ are shown in Figs. 4(a-c). At the lowest prestress, $\sigma=3.5 \mathrm{GPa}$ (Fig. 4(a)), the crack exhibited very little growth (from $l_{\mathrm{o}}=5.7 \mathrm{~nm}$ nucleation length to $l \approx 10 \mathrm{~nm}$ equilibrium length). The equilibrium was reached due to the formation of the two nanotwins at the tip (2) in Fig. 4(a)), propagating in the $-x$ direction, which plastically relieved enough strain energy to arrest the crack growth. At $\sigma=4.0 \mathrm{GPa}$ (Fig. 4(b)), the growth process is very similar to the case of $\sigma=$ 
Invited paper for the Special Issue of Journal of Materials Science Special Issue Title: "Nanostructured Materials and Mechanical Behavior"

3.75 GPa, discussed earlier. In the $-x$ direction, the crack is blunted by deformation twins, growing symmetrically in the two crystals, while in the $+x$ direction, the crack is arrested by a dislocation burst. Increasing the prestress to $\sigma=4.25 \mathrm{GPa}$ resulted in a substantial increase of the crack length (Fig. 4(c)), accompanied by more intensive plastic processes such as enhanced deformation twinning (2) in Fig. 4(c)) and enhanced dislocation activity (3) and (5) in Fig. 4(c)). A more detailed description and analysis of the atomistic processes taking place at the two tips of the growing crack is given in ref. [28]. The present discussion will focus on the dynamics of the crack growth and the energy transformations at the crack tips which define the crack growth resistance.

The speed of crack growth can be estimated in the MD simulation by determining the rate of increase of the crack surface area. This can be done by using the identification procedure for "surface" atoms as described in Section 2. On average, $1 \mathrm{~nm}^{2}$ of a flat $\left\{\begin{array}{l}5 \\ 57\end{array}\right\}$ surface contains 16 surface atoms. The crack free surface $S$ is estimated by counting the number of surface atoms, and when divided by $2 h$ (see Fig. 1 , the prefactor 2 accounts for the two crack surfaces), conveniently gives an effective crack length $l$.

The dependence of $l$ with time, given in Fig. 5, shows distinctively different behavior for the four prestress values used in the study. At the lowest prestress, $\sigma=3.5 \mathrm{GPa}$, the crack shows a slow, but steady growth at the speed of $25 \mathrm{~m} / \mathrm{s}$ for almost $150 \mathrm{ps}$ and then arrests (Fig. 5). The reason for this arrest is the formation of two symmetrical nanotwins at the crack tip propagating in the $-x$ direction (Fig. 4(a)), in the same way as in the $\sigma=3.75 \mathrm{GPa}$ case, discussed previously (Fig. 3). These nanotwins relieve the stress around the crack [28] and prevent its further growth. The crack reaches an elasto-plastic equilibrium with the surrounding material at a length of 10 nm (Fig. 4(a)). 
Invited paper for the Special Issue of Journal of Materials Science Special Issue Title: "Nanostructured Materials and Mechanical Behavior"

At a slightly higher prestress of $\sigma=3.75 \mathrm{GPa}$, the average speed of crack growth increases dramatically from 25 to $511 \mathrm{~m} / \mathrm{s}$ (see the corresponding curve in Fig. 5). This fast growth takes place in the $+x$ direction only, while the propagation in the $-x$ direction is blunted by the active formation of the two symmetric nanotwins (2) in Fig. 3). After reaching a length of $35 \mathrm{~nm}$, the crack growth in the $+x$ direction is arrested, as shown in Fig. 5, by the emission of several dislocations (seen as (5) Fig. 3(i)). The stress is relieved and a smoothing of the crack free surface, initially rough because of the void coalescent mechanism of growth (4) in Figs. 3(d-i)), begins to take place resulting in a slight decrease of the surface area. Because the crack length is being measured through the crack free surface area, as described previously, this relaxation of the surface gives an effective "shortening" of the crack, producing a region of apparent negative growth after the crack length has reached its peak at $t=60 \mathrm{ps}$ in Fig. 5. The distance between the two crack tips does not change in the process, indicating that the apparent "shortening" is not a result of crack closure, but only due to surface relaxation effects.

Qualitatively, very similar behavior is observed for the prestress of $4.0 \mathrm{GPa}$ (see the corresponding curve in Fig. 5). The average crack speed has increased to $701 \mathrm{~m} / \mathrm{s}$ and the crack has stopped its growth at almost the same length of $35 \mathrm{~nm}$ (Fig. 4(b)), but earlier in time compared to the case of $3.75 \mathrm{GPa}$ prestress. The region of the follow up negative growth for $\sigma=$ 4.0 $\mathrm{GPa}$ is considerably deeper than for $\sigma=3.75 \mathrm{GPa}$, indicating more intensive relaxation - a result of more intensive void formation and plastic processes accompanying the faster crack growth.

When the prestress is increased up to $4.25 \mathrm{GPa}$ another substantial change in the crack behavior is observed (see the corresponding curve in Fig. 5). Unlike simulations at lower values of prestress, the crack growth at $\sigma=4.25 \mathrm{GPa}$ occurs in three discrete increments. The first 
Invited paper for the Special Issue of Journal of Materials Science Special Issue Title: "Nanostructured Materials and Mechanical Behavior"

increment occurs from $0<t<25 \mathrm{ps}$ at an average speed of $752 \mathrm{~m} / \mathrm{s}$, while the second increment occurs from $75<t<100 \mathrm{ps}$ at an average speed of $687 \mathrm{~m} / \mathrm{s}$, and the third at $125<t<155 \mathrm{ps}$ at an average speed of $651 \mathrm{~m} / \mathrm{s}$. These repeating cycles of resumed growth can also be observed on the velocity vs. time curve shown in Fig. 6(a).

A detailed analysis [34] revealed that the observed oscillatory behavior of the crack growth is a result of a dynamic instability $[35,36]$ developed at the crack tip each time the instantaneous propagating velocity of the tip reaches a critical value $v_{\mathrm{c}}$ of approximately $35 \%$ of the Rayleigh wave speed $c_{R}=3180 \mathrm{~m} / \mathrm{s}$ for this model. This instability was shown [34] to create a series of periodic dislocation bursts that appeared in the simulation shortly after the crack tip velocity reached $v_{\mathrm{c}}$. These dislocation bursts (some of which are still seen as (5) in Fig. 4(c)) reduce the crack propagation velocity. It is possible that the same mechanism of dislocation bursts is responsible for stopping the crack growth in the cases of $\sigma=3.75 \mathrm{GPa}$ and $\sigma=4.0 \mathrm{GPa}$, where the tip velocity reached a peak of approximately $0.3 c_{R}$ and $0.4 c_{R}$, respectively $($ Fig. $6(\mathrm{~b}, \mathrm{c})$ ). The values of $0.3 c_{R}$ and $0.4 c_{R}$ may have been sufficient to induce dynamic instability and trigger the observed subsequent dislocation emission (5) in Fig. 3(i) and Fig. 4(b), respectively), thus blunting the crack tip in the $+x$ direction for both cases. In the case of $4.25 \mathrm{GPa}$ prestress, the accumulated strain energy in the system was sufficient to overcome this dynamic blunting and restore the crack growth on two successive occasions, producing the velocity oscillations observed in Fig. 6(a).

Another important factor in the process of fast intergranular crack growth is the role of the GB. When dynamic instability occurs, the GB interface prevents crack branching - a typical phenomenon in brittle fracture [35,36], making it possible for the crack to restore its growth when the propagation speed decreased below $v_{\mathrm{c}}$. Consequently, the crack can overcome the 
Invited paper for the Special Issue of Journal of Materials Science

Special Issue Title: "Nanostructured Materials and Mechanical Behavior"

dynamics instability and continue its growth if the system has a sufficient amount of strain energy. The case which is observed for the $\sigma=4.25$ GPa prestress (as seen in Fig. 4(c) and Fig.

5).

\section{Energy release mechanisms during crack growth}

The process of crack growth, as described in Section 2, occurs in a prestrained system under fixed displacement boundary conditions. During crack growth, there is no external work done on the system and the system exchanges energy only in the form of heat transfer through the applied Nose-Hoover thermostat [20]. The crack grows by expending elastic energy that was stored when the system was initially prestrained. The energy balance can be expressed as

$$
\Delta E_{e l}=E_{s}-\Delta E_{G B}+E_{p l}+\Delta Q
$$

where: $\Delta E_{e l}$ is the decrease of the stored elastic energy as the crack grows; $E_{s}=2 l \gamma_{\mathrm{s}}$, is the energy of the newly formed free surface of the crack; $\Delta E_{G B}=l \gamma_{\mathrm{GB}}$, is the excess (i.e., above the perfect crystal) energy of the destroyed GB interface; $E_{p l}$ is the energy related to all plastic processes taking place in the plastic zone around the crack tips; and $\Delta Q$ is the heat released in the process.

By summing the energies from every atom in each of the structural states discussed in Section 2, it is possible to determine each structural state's contribution to the potential energy of the system (e.g., free surface energy, GB energy, elastic energy and plastic energy). The total free surface energy, $E_{s}$ in Eq. (7), is determined as the sum of the potential energy of all surface atoms (as defined in Section 2). The GB energy $E_{G B}$ is approximated as the sum of the potential energy of all disordered atoms. The computed change of the grain boundary energy, $-\Delta E_{G B}$ in Eq. (7), is negative since the overall number of GB atoms decreases due to the transformation of 
Invited paper for the Special Issue of Journal of Materials Science Special Issue Title: "Nanostructured Materials and Mechanical Behavior"

GB atoms into surface or crystalline atoms as the crack grows. Note that this calculation of grain boundary energy is approximate since a small number of disordered atoms are also present as either dislocation cores or vacancies.

The elastic energy, $E_{e l}$ in Eq. (7), is determined as the excess energy of the atoms in a fcc state, as identified by the CNA technique [29]. Here, the atoms in the fcc state form the elastically deformed crystal lattice, and their excess potential energy is the elastic energy of the lattice. Plastic deformation occurs mainly through the formation of twins and stacking faults as a result of dislocation nucleation. Since twins and stacking faults are comprised of hcp atoms [32], the plastic energy, $E_{p l}$ in Eq. (7), is determined as the sum of the excess potential energy of all the hcp atoms in the system. However, this energy is only a part of the total energy consumed by the plastic processes. Another part, for example, the energy required to drive a dislocation, is transferred into heat and contributes to the $\Delta Q$ term in Eq. (7).

The heat generated during crack growth is taken out of the system by the MD thermostat and by the absorbing layers as discussed in Section 2. The Nose-Hoover MD thermostat [20] introduces external non-conservative forces to the atoms decreasing or increasing their kinetic energy to keep the overall temperature constant. The work done by these non-conservative forces and by the damping forces applied to the atoms in the absorbing layers results in heat release of the system and can be calculated during the simulation to get the term $\Delta Q$ in Eq. (7).

Despite its convenience and relatively easy implementation, the energy identification procedure outlined in the previous paragraphs, has some deficiencies. First; the precision of the identification depends ultimately on the precision of the CNA and the surface atoms identification techniques. The precision of both of these techniques depends on the ability to distinguish between the first and second nearest neighbors of a given atom. This distinction is 
Invited paper for the Special Issue of Journal of Materials Science Special Issue Title: "Nanostructured Materials and Mechanical Behavior"

generally based on the distance between the neighboring atoms and is most appropriate when the temperature is relatively low (at room temperature and below when the thermal atomic vibrations are low), and when the elastic strain is relatively small (on the order of a few percent, so that the crystal lattice is not overly deformed). Second; the actual energetic contributions of each of the structural states is not as clearly delineated as this method suggests. For example, part of the potential energy of a non-fcc atom should also be included in the elastic energy, as the non-fcc structures also have elastic energy. Nevertheless, the energy to create such a structural defect in a perfect lattice (the excess energy) is usually much higher than the elastic energy of the formation", thus latter can be neglected. Considering the issues outlined above, the suggested procedure in sorting out the different energy contributions in the process of crack growth is a good trade-off between simplicity, effectiveness and precision.

Under the prestrained conditions of the model (Fig. 1), the stored elastic energy is the only source of energy for the crack to grow. The decrease of the elastic energy for a growing crack for each of the four applied prestress conditions is shown in Fig. 7. For comparison, FEM calculations for quasistatic crack growth in a pure elastic (no plasticity) material with the same elastic coefficients as those derived from the EAM interatomic potential [19] are given. In all cases, the MD energy curves fall below the FEM lines, indicating a faster release of the stored elastic energy than is expected for the quasistatic crack growth in the absence of plasticity. For the cases of 3.75 and 4.0 GPa prestress, the majority of energy release occurs at the end of the crack growth when the crack is blunted by a dislocation burst (see (5) in Fig. 3(i) and Fig. 4(b)). The process is accompanied by relaxation and smoothing of the initially rough crack surfaces,

\footnotetext{
* For example, the formation energy of a vacancy for the interatomic potential used in this study [19] is $0.64 \div 0.68$ $\mathrm{eV} /$ atom, while the strain energy for $1 \%$ strain is only $0.001 \mathrm{eV} /$ atom.
} 
Invited paper for the Special Issue of Journal of Materials Science Special Issue Title: "Nanostructured Materials and Mechanical Behavior"

when the crack becomes stationary, producing an effective shortening of the crack length as seen in Fig. 7 and Fig. 5.

For the case of $4.25 \mathrm{GPa}$, there are three noticeable localized drops in the energy curve at 28 $\mathrm{nm}, 45 \mathrm{~nm}$ and $60 \mathrm{~nm}$. These instantaneous decreases in the stored elastic energy coincide with the time of the three successive peaks in the crack speed, shown in Fig. 6(a), when the critical velocity is reached. At this velocity, the fast propagating crack tip becomes dynamically unstable and produces dislocation bursts [34]. The observed (see Fig. 7) drops in the stored elastic energy for $\sigma=4.25 \mathrm{GPa}$ case are a direct result of the dynamically initiated dislocation bursts.

As the crack grows, the elastic energy plus the energy of the destroyed GB is transferred into surface energy, plastic energy and heat (Eq. (7)). A plot of each of the energy contributions in Eq. (7), coming from different processes during crack growth are shown in Fig. 8. For the three given prestresses of $\sigma=3.75,4.0$ and $4.25 \mathrm{GPa}$ (in the case of $\sigma=3.5 \mathrm{GPA}$ the growth of the crack was insufficient to reliably determine the energy), the largest part of the energy was released in the form of heat. The next most substantial amount of energy was expended creating the free surface. A small amount of energy was recovered by destroying the GB. The potential energy of the hep atoms (given in Fig. 8) can be related to the stored plastic energy $E_{p l}$ in Eq. (7) as it gives the energy of formation for the twin boundaries and the stacking faults produced in the process of crack growth (seen in Figs 3 and 4). Fig. 8 shows that the energy associated with the hcp atoms is small compared to the other forms of energy release. The greatest component of the energy released in the plastic processes does not go into the creation of these defects, but into viscous heat dissipation as defects are swept away from the crack tip. Another contribution to the heat release, shown in Fig. 8, comes from the phonons produced in the process of interface debonding. Since the heat release associated with phonons was not specifically determined, the 
Invited paper for the Special Issue of Journal of Materials Science Special Issue Title: "Nanostructured Materials and Mechanical Behavior"

curves for the heat release in Fig. 8 reflect the total amount of heat of all dissipative processes in the system.

The slope of the energy release curves, shown in Fig. 8, gives the energy release rate. Fig. 9 compares the energy release rates of the heat release, free surface formation, and GB destruction for the four average initial crack tip velocities (estimated in Fig. 5) obtained at the four prestresses. As expected, the energy release rates for surface creation and GB destruction do not depend on the crack propagation speed. These rates (see Fig. 8) are close to twice the surface energy per unit area $\gamma_{s}$, and to the GB energy per unit area $\gamma_{G B}$, respectively $\left(\gamma_{s} \approx 1 \mathrm{~J} / \mathrm{m}^{2}[19]\right.$ and $\left.\gamma_{G B} \approx 0.65 \mathrm{~J} / \mathrm{m}^{2}[34]\right)$. In fact, $\gamma_{s}$ extracted from Fig. 9 is slightly higher than $1.0 \mathrm{~J} / \mathrm{m}^{2}$, which can be attributed to the non-relaxed state of the crack free surface. The GB energy released per unit area $\gamma_{G B}$ also deviates slightly from the static value of $0.65 \mathrm{~J} / \mathrm{m}^{2}$, and tends to decrease at high crack propagation speeds (above $700 \mathrm{~m} / \mathrm{s}$ ). As $\gamma_{G B}$ should not depend on the speed of the crack propagation, the only conceivable explanation appears to be that the decrease of the disordered atoms, belonging to the GB, is partly compensated by the appearance of more disordered atoms via the formation of dislocations at high crack tip velocity (recall that the GB energy is defined as the energy of the disordered atoms, assuming that most of them belong to the GB interface).

Unlike the energy release rate spent to create the crack free surface, the rate of heat release increases rapidly with increasing crack propagation speed (Fig. 9). While at the lowest simulated speed of $25 \mathrm{~m} / \mathrm{s}$, the heat release rate is lower than the surface energy release rate, at high speed it becomes the dominant contribution to energy release rate. Initially, $G_{h e a t}$ increases linearly with the crack propagation speed, suggesting viscous crack propagation. At high velocities $G_{\text {heat }}$ increases faster than linear, which is consistent with both the theoretical basis for the MD 
Invited paper for the Special Issue of Journal of Materials Science

Special Issue Title: "Nanostructured Materials and Mechanical Behavior"

simulation model [37] and experimental observations of a crack moving in a brittle amorphous material [38].

\section{Conclusions}

Using a molecular-dynamics model for crack propagation under steady-state conditions, the presented paper studies the dynamics and energetics of intergranular crack growth along a flat grain boundary in aluminum. The crack is initiated by screening the interaction forces between atoms in a short region on both sides of the grain-boundary interface in a hydrostatically prestressed bicrystalline aluminum plate. The dynamics of the crack shows a strong non-linear dependence of the speed of the crack propagation on the prestress. When the crack propagation speed increases to $0.35 \%$ of the Rayleigh wave speed, a dynamic instability occurs at the crack tip producing periodic dislocation bursts until the crack is blunted. The GB interface prevents crack branching.

Using the ability of the molecular-dynamics simulation to identify atoms in different structural configurations, it was possible to identify the energy contribution of different processes taking place during crack growth. The energy contributions were determined as: (i) elastic energy - defined as the excess potential energy of the atoms in fcc crystallographic state; (ii) plastically stored energy - the excess energy of stacking faults and twin boundaries; (iii) grain-boundary energy and (iv) surface energy. Additionally, the energy dissipated as heat in the system was determined by monitoring the heat exchange through the thermostat. This energetic

analysis indicates that the majority of energy in a fast growing crack is dissipated as heat. This dissipation increases in a linear fashion at low speed and faster than linear at a speed approaching $1 / 3$ the Rayleigh wave speed. At relatively low speed (below $200 \mathrm{~m} / \mathrm{s}$ or less than few percent of 
Invited paper for the Special Issue of Journal of Materials Science

Special Issue Title: "Nanostructured Materials and Mechanical Behavior"

the Rayleigh wave speed), the dissipated heat becomes lower than the energy needed to create the crack free surface.

\section{Acknowledgements}

V. Yamakov and D. R. Phillips were sponsored through cooperative agreement NCC-102043 with the National Institute of Aerospace and contract NAS1-00135 with Lockheed Martin Space Operations, respectively. 
Invited paper for the Special Issue of Journal of Materials Science

Special Issue Title: "Nanostructured Materials and Mechanical Behavior"

\section{References:}

1. K. S. KUMAR, S. SURESH, M. F. CHISHOLM, J. A. HORTON and P. WANG, Acta Mater. 51 (2003) 387.

2. D. WOlf, V. YAMAKOV, S. R. PHILlPOT, A. K. MUKHERJEE and H. GLEITER, Acta Mater. (2005) 53, 1.

3. A. A. GRIFFITH, Philos. Trans. Royal Soc. London A221 (1920) 163.

4. E. OROWAN, Transactions of the Institute of Engineers and Shipbuilders Scotland 89 (1945) 165.

5. G. R. IRWIN, In: F. Jonassen, W. P. Roop, R. T. Bayless (Eds.), American Society of Metals, Cleveland (1949) 147.

6. J. W. KYSAR, J. Mech. Phys. Solids 51 (2003) 795.

7. D. WOLF, J. Mater. Res. (1990) 5, 1708.

8. R. G. HOAGLAND, Philos. Mag. A 76 (1997) 543.

9. F. CLERI, S. R. PHILLPOT and D. WOLF, Interface Sci. 7 (1999) 45.

10. D. FARKAS, Philos. Mag. A 80 (2000) 1425.

11. D. FARKAS, H. VAN SWYGENHOVEN and P. M. DERLET, Phys. Rev. B 66 (2002) 060101.

12. R. E. RUDD and J. F. BELAK, Comp. Mater. Sci. 24 (2002) 148.

13. L. GOLUBOVIC, A. PEREDERA and M GOLUBOVIC, Phys. Rev. E 52 (1995) 4640.

14. M. GRUJICIC, H. ZHAO and G. L. KRASKO, Int. J. Refract. Metals Hard. Mater. 15 (1997) 341.

15. P. GUMBSCH, S. J. ZHOU and B. L. HOLIAN, Phys. Rev. B 55 (1997) 3445.

16. P. GUMBSCH, Mat. Sci. Eng. A 260 (1999) 72.

17. P. HEINO, H. HÄKKINEN and K. KASKI, Phys. Rev. B 58 (1998) 641.

18. J. STAMPFL and O. KOLEDNIK, Int. J. Fracture 101 (2000) 321.

19. Y. MISHIN, D. FARKAS, M. J. MEHL and D. A. PAPACONSTANTOPOULOS, Phys. Rev. B 59 (1999) 3393. 
Invited paper for the Special Issue of Journal of Materials Science

Special Issue Title: "Nanostructured Materials and Mechanical Behavior"

20. S. NOSE, J. Chem. Phys. 81 (1984) 511.

21. M. BARBER, J. DONLEY and J. S. LANGER, Phys. Rev. A 40 (1989) 366.

22. U. DAHMEN, J. D. HeTheRINGTON, M. A. O'KeEFE, K. H. WESTMACOTT, M. J. MILLS, M. S. DAW and V. VITEK, Philos. Mag. Lett. 62 (1990) 327.

23. E. B. TADMOR and S. HAI, J. Mech. Phys. Solids 51 (2003) 765.

24. V. YAMAKOV, D. WOLF, S. R. PHILLPOT and H. GLEITER, Nature Mater. 1 (2002) 45.

25. V. YAMAKOV, D. WOLF, S. R. PHILLPOT and H. GLEITER, Acta Mater. 51 (2003) 4135.

26. V. YAMAKOV, D. WOLF, S. R. PHILLPOT, A. K. MUKHERJEE and H. GLEITER, Philos. Mag. Lett. 83 (2003) 385.

27. M. PARRINELlO, A. RAHMAN, J. Appl. Phys. 52 (1981) 7182.

28. V. YAMAKOV, E. SAETHER, D. R. PHILliPS and E. H. GLAESSGEN, J. Mech. Phys. solids (in press).

29. J. D. HONEYCUTT and H. C. ANDERSEN, J. Phys. Chem. 91 (1987) 4950.

30. A. S. CLARKE and H. JONSSON, Phys. Rev. E 47 (1993) 3975.

31. J. WEERTMAN and J. R. WEERTMAN, Elementary dislocation theory. (Oxford University Press, New York, 1992).

32. J. SCHIOTZ, T. VEGGE, F. D. DI TOLLA and K. W. JACOBSEN, Phys. Rev. B 60 (1999) 11971.

33. J. R. RICE, 1992. J. Mech. Phys. Solids 40 (1992) 239.

34. V. YAMAKOV, E. SAETHER, D. R. PHILliPS and E. H. GLAESSGEN, Phys. Rev. Lett. 95 (2005) 015502.

35. F. F. ABRAHAM, D. BRODBECK, R. A. RAFEY and W. E. RUDGE, Phys. Rev. Lett. 73 (1994) 272.

36. F. F. ABRAHAM, J. Mech. Phys. Solids 49 (2001) 2095.

37. E. S. C. CHING, Phys. Rev. E 49 (1994) 3382.

38. E. SHARON, S. P. GROSS and J. FINEBERG, Phys. Rev. Lett. 76 (1996) 2117. 
Invited paper for the Special Issue of Journal of Materials Science

Special Issue Title: "Nanostructured Materials and Mechanical Behavior"

\section{Figures:}

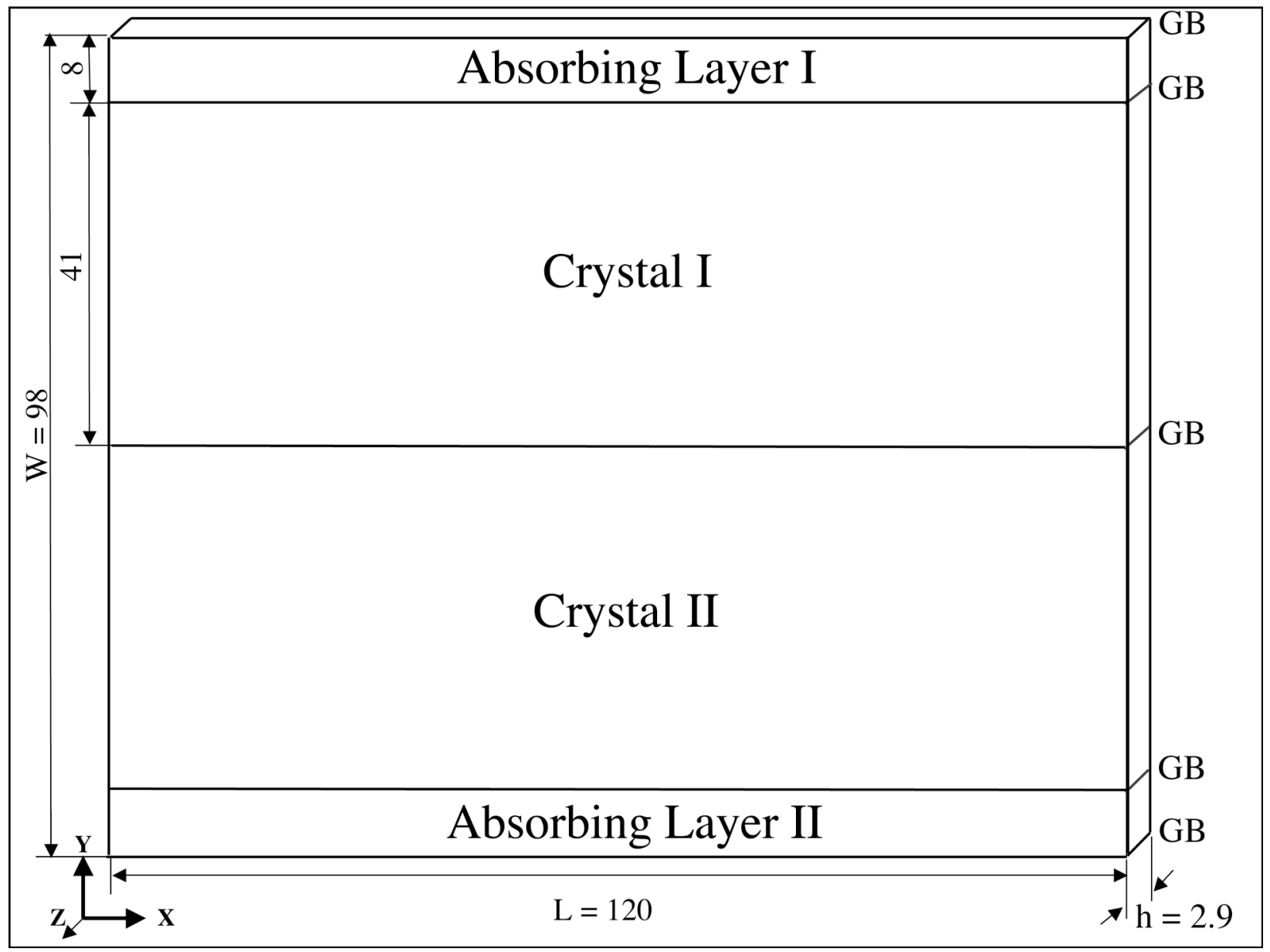

Fig. 1. Schematic representation of the simulation model, explained in the text. Size dimensions are in $\mathrm{nm}$. 
Invited paper for the Special Issue of Journal of Materials Science

Special Issue Title: "Nanostructured Materials and Mechanical Behavior"

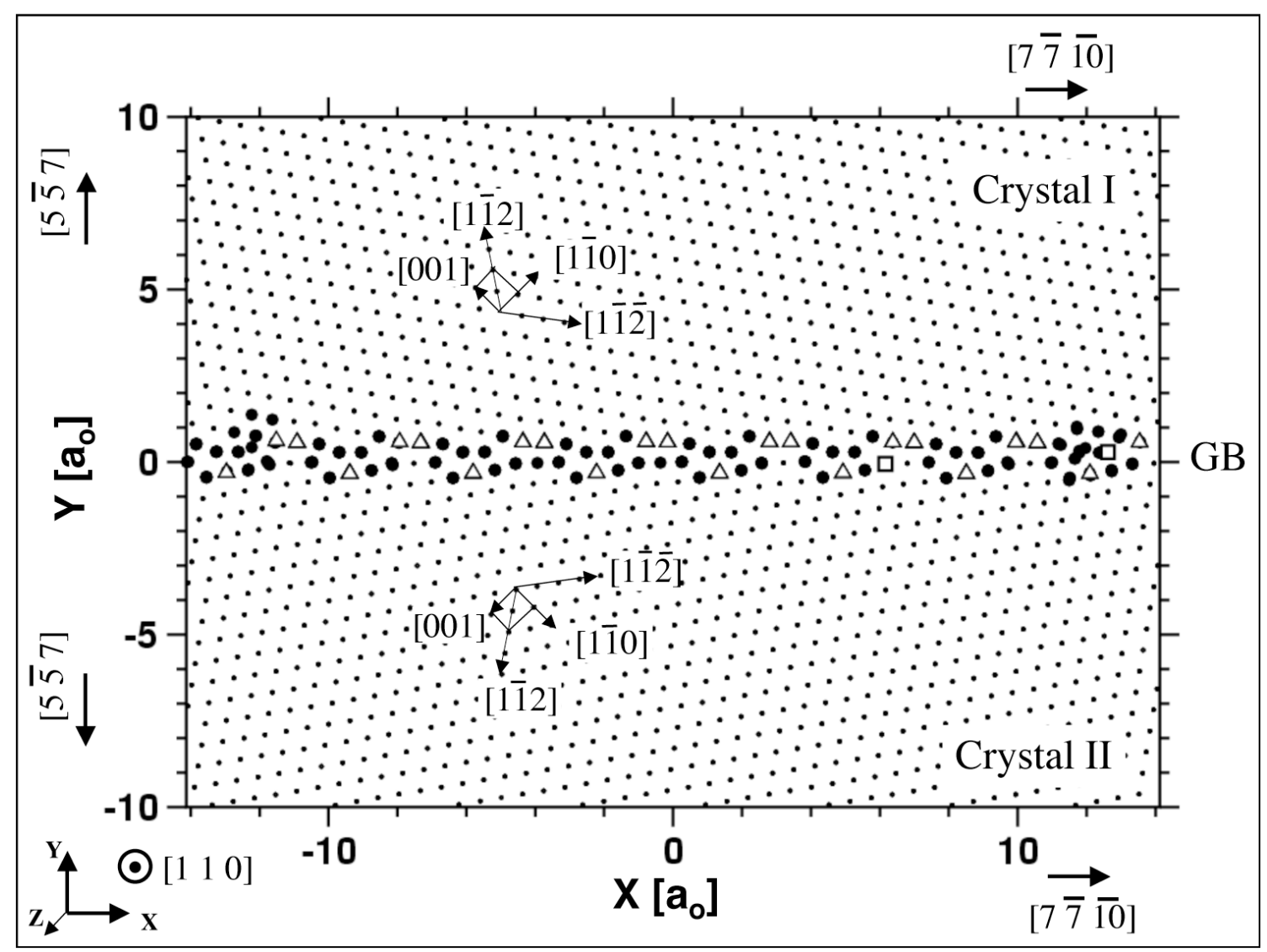

Fig. 2. Atomistic snapshot giving the crystallography and structure of the GB interface. Common neighbor analysis (CNA) $[29,30]$ is used to identify atoms in different crystallographic states: fcc (small dots), hcp (triangles), and non-crystalline atoms (large dots). Atoms with more than 1/3 of their nearest neighbors missing are identified as surface atoms (squares), indicating existing vacancies in the GB. The length scale is in units of the lattice constant of $\mathrm{Al}, \mathrm{a}_{\mathrm{o}}=0.405 \mathrm{~nm}$. 
Invited paper for the Special Issue of Journal of Materials Science

Special Issue Title: "Nanostructured Materials and Mechanical Behavior"
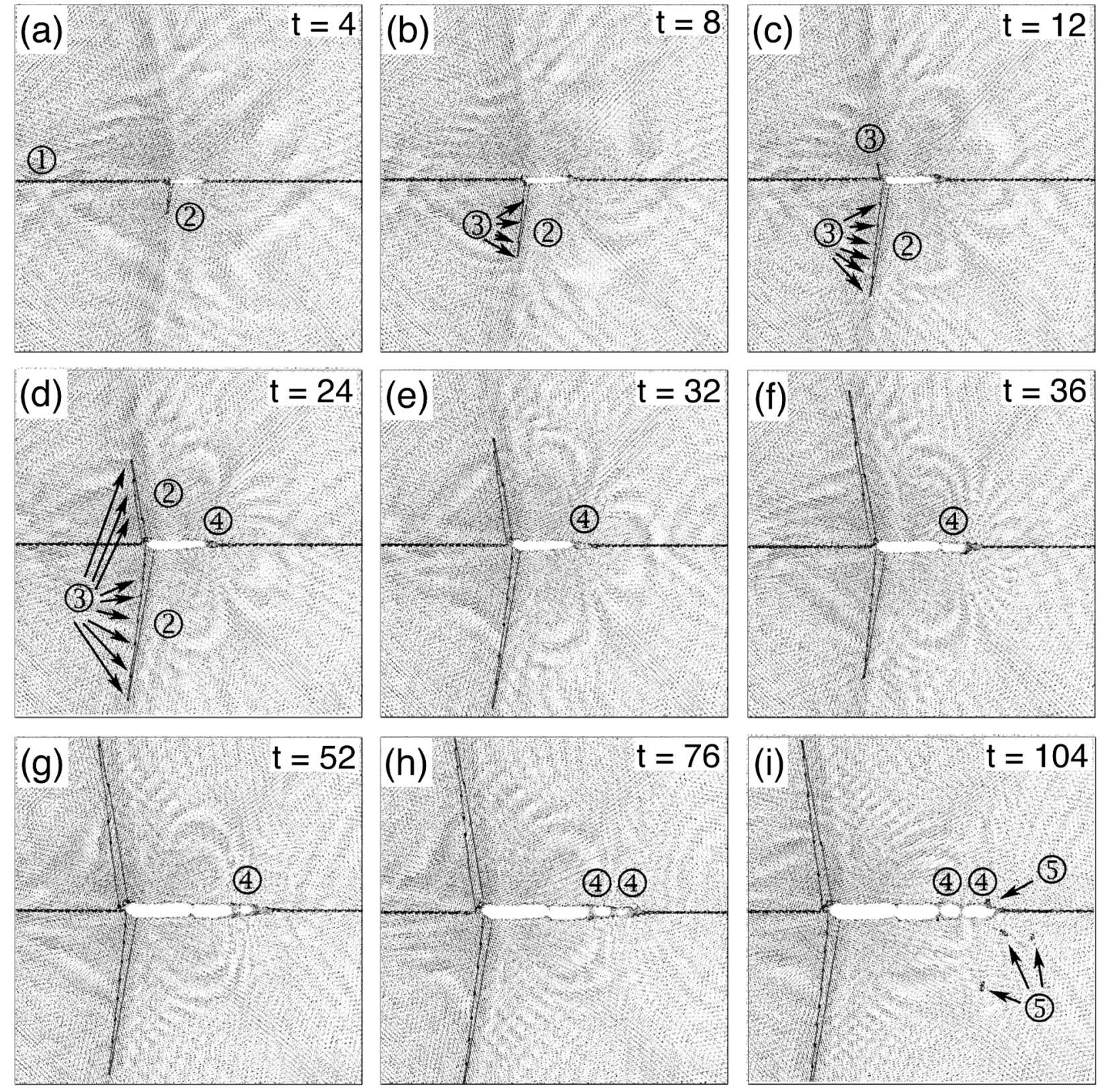

Fig. 3. A series of snapshots monitoring the process of crack growth in the MD system prestressed at $3.75 \mathrm{GPa}$ tensile hydrostatic load. The snapshots are taken at various times from the crack initiation, $t$, given in ps. CNA is used to identify atoms in hep crystallographic state (forming the grey lines of twin boundaries and stacking faults), and non-crystalline atoms (in black indicating the GB and dislocation cores). Thus, a number of different formations are indicated in the figure as follows: (1) - GB interface; (2) - deformation twin; (3) - partial or twinning dislocation; (4) - nanovoid at the crack tip; (5) - slip dislocation. The wavy Moire patterns in the crystal regions make visible the strain patterns and phonon waves in the crystal (see text). 
Invited paper for the Special Issue of Journal of Materials Science

Special Issue Title: "Nanostructured Materials and Mechanical Behavior"
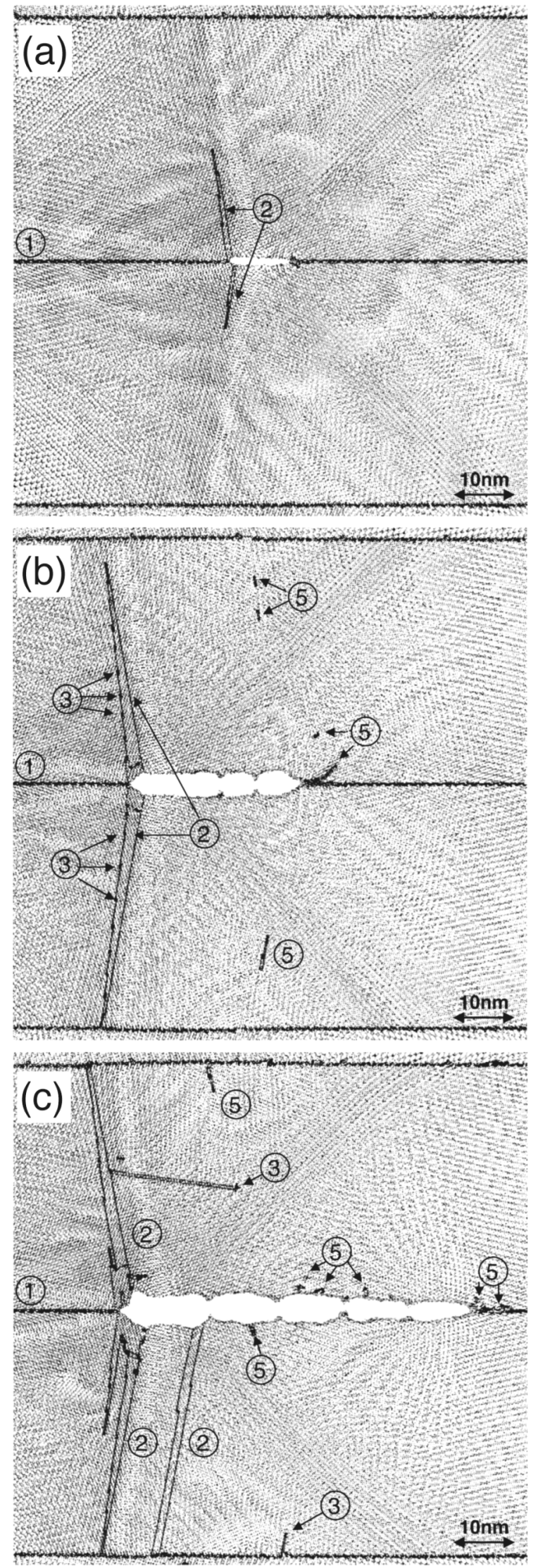

Fig. 4. MD snapshots of cracks, which have propagated in the MD system, shown in Fig. 1, prestressed at three different initial hydrostatic stresses: $\sigma=3.5$ (a), 4.0 (b) and $4.25 \mathrm{GPa}$ (c). As in Fig. 3, CNA is used to identify atoms in different formations (1)-(5), which correspond to the types indicated in Fig. 3. 


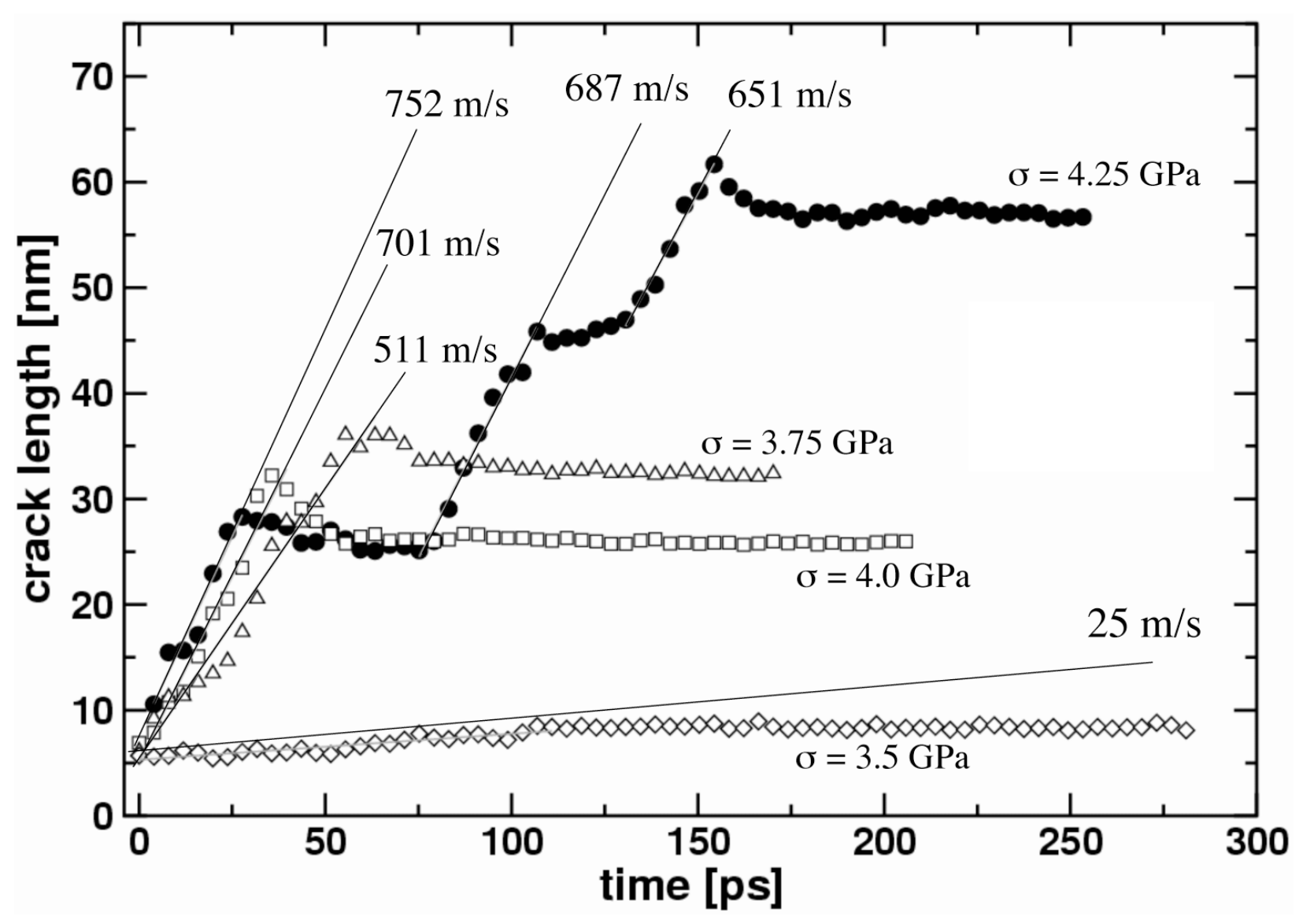

Fig. 5. Crack length vs. time for the four prestress values applied in the simulation. 

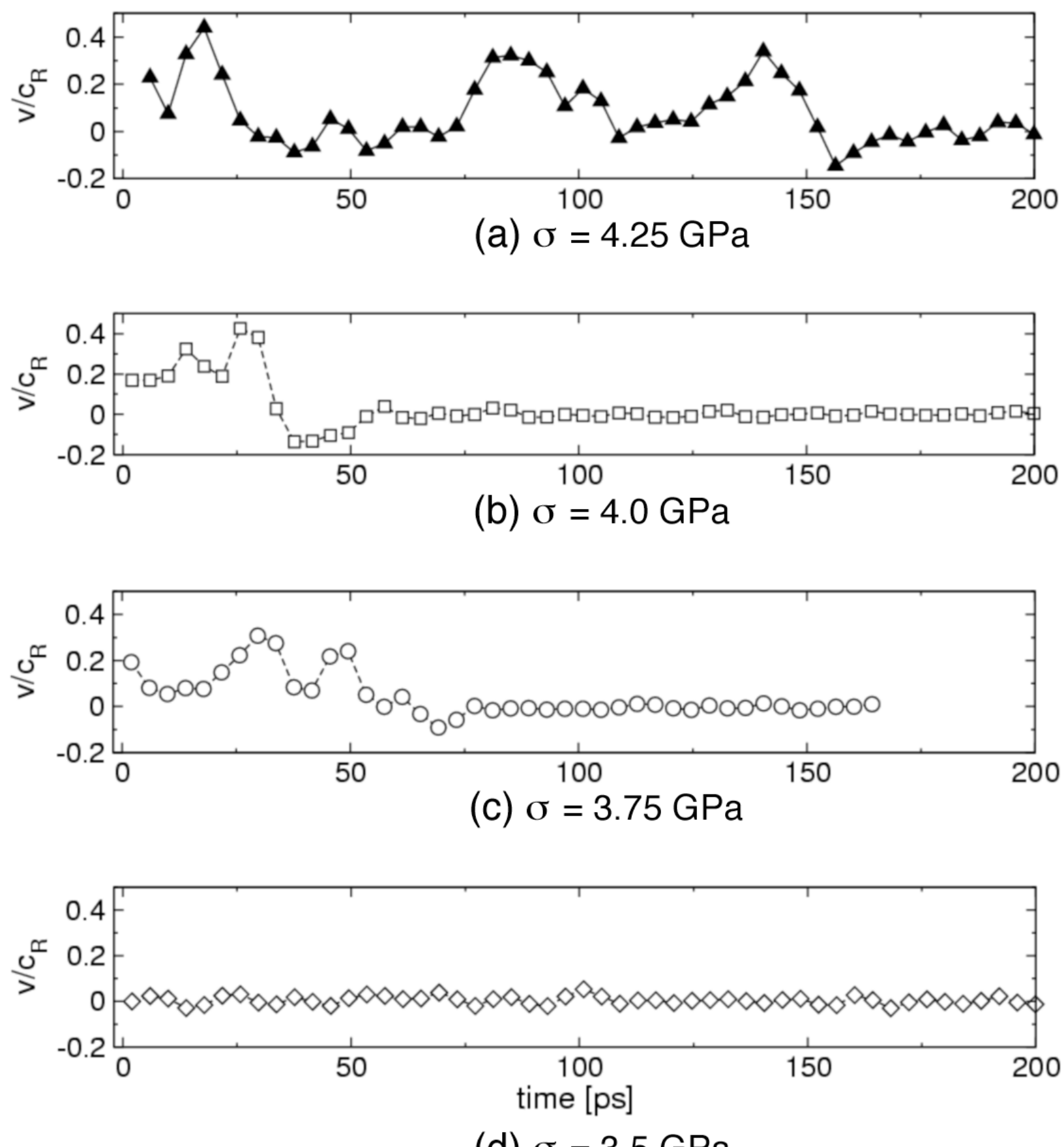

Fig. 6. The speed of crack growth in units of the Rayleigh wave speed, $c_{R}=3180 \mathrm{~m} / \mathrm{s}$, vs. time for the four prestress values applied in the simulation. 


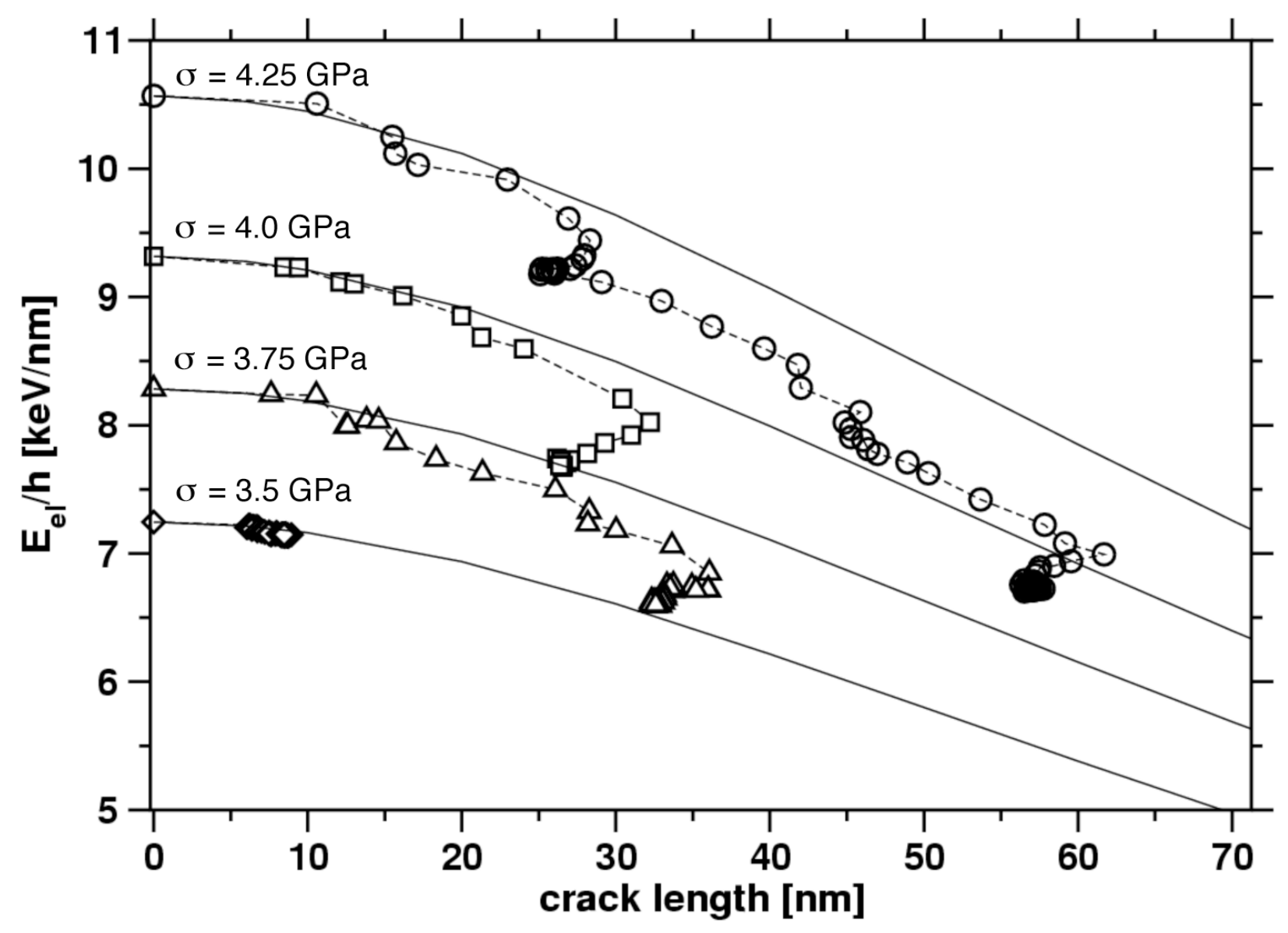

Fig. 7. The stored elastic energy in the simulated system vs. the length of the growing crack. Symbols represent MD simulation results. The solid lines represent FEM quasistatic elastic simulation results. 


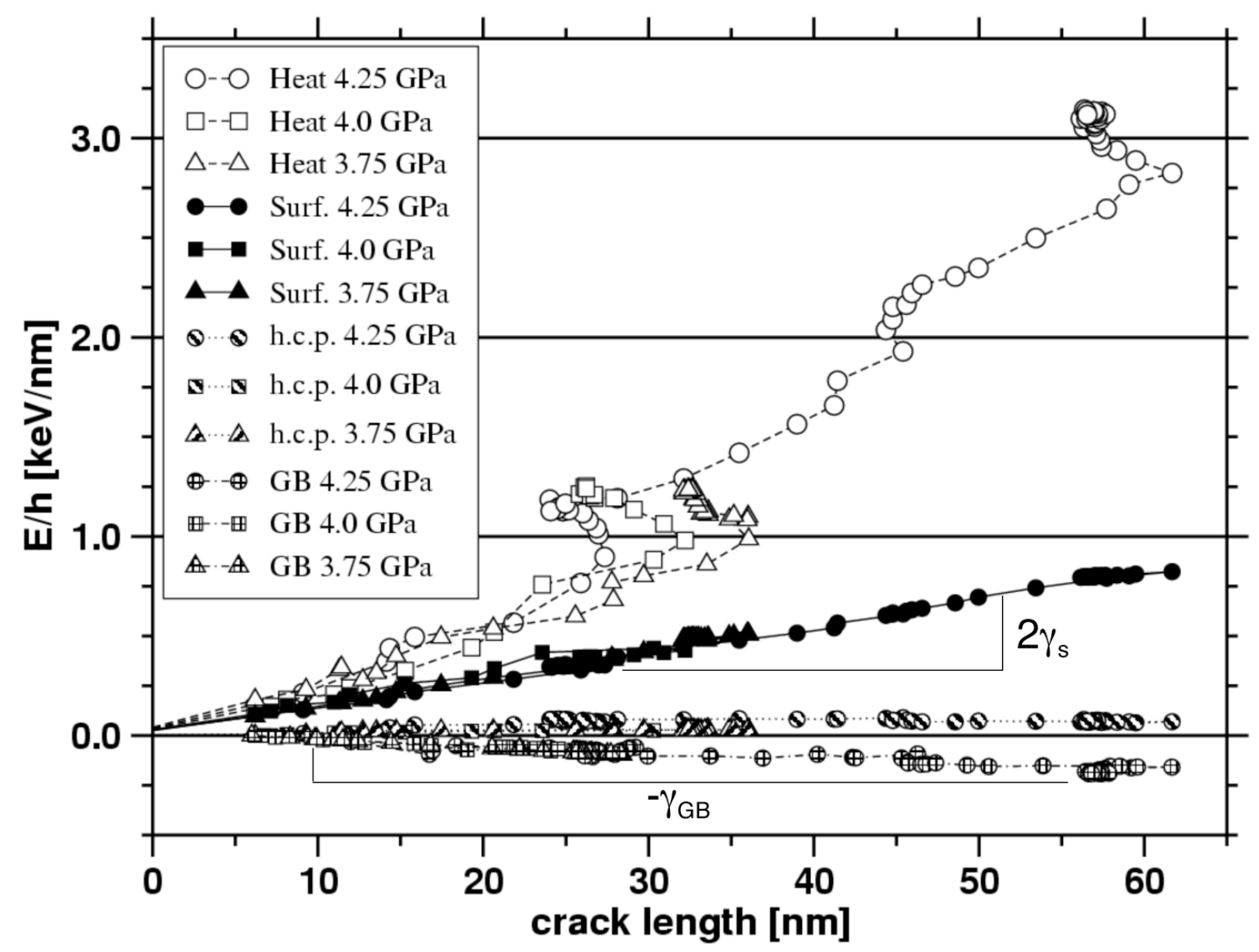

Fig. 8. The energy released through four different mechanisms accompanying the crack growth: heat dissipation, free surface formation, growth of stacking faults and twin boundaries, and the energy gained by destroying the GB interface. 


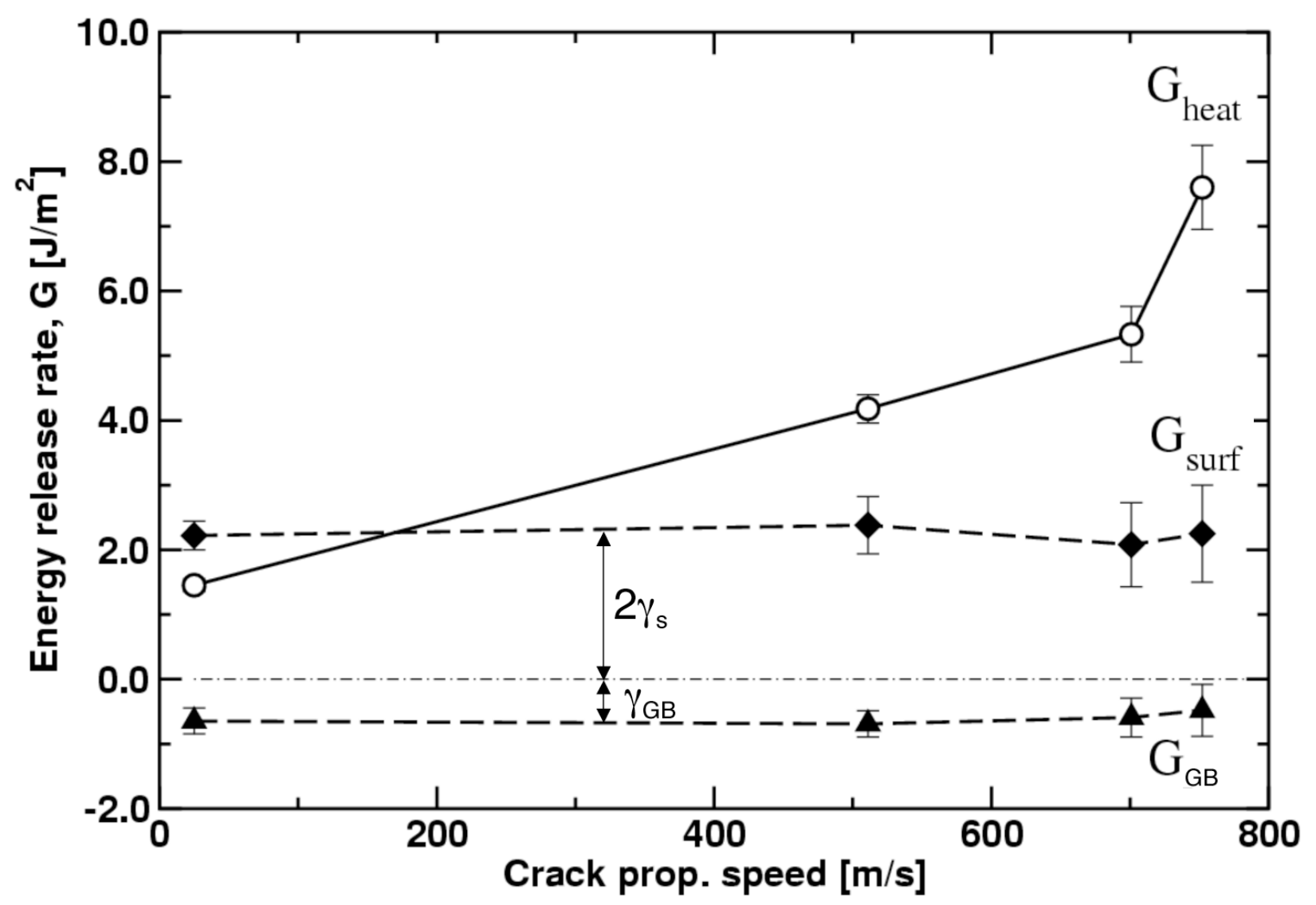

Fig. 9. The energy release rate for three different mechanisms accompanying the crack growth: heat dissipation, free surface formation, and the rate of energy gained by destroying the GB interface. 